\title{
Clinical presentation and management of proatlas segmentation defect presenting with palatal myoclonus: case report
}

\author{
Raheel Ahmed, MD, PhD, and Arnold H. Menezes, MD \\ Department of Neurosurgery, University of lowa Hospitals and Clinics, lowa City, lowa \\ Clinical presentation of craniovertebral junction disorders may range from acute catastrophic neurological deficits to \\ insidious signs and symptoms that may mask the underlying etiology. Prompt recognition and treatment is essential to \\ avert long-term neurological morbidity. Proatlas segmentation disorders are a rare group of developmental disorders \\ involving the craniocervical junction. Abnormal bony segmentation leads to malformed bony structures that can in turn \\ lead to neurological deficits through bony compression of the cervicomedullary junction. This report details a proatlas \\ segmentation defect presenting as palatal myoclonus, a rare movement disorder. The clinical presentation, surgical \\ management, and neuroanatomical basis for the disorder is presented. This report highlights the myriad clinical presen- \\ tations of craniovertebral disorders and emphasizes a rare but treatable etiology for palatal myoclonus. \\ http://thejns.org/doi/abs/10.3171/2015.1.PEDS14671
}

KEY WORDS proatlas segmentation anomaly; symptomatic palatal tremor; palatal myoclonus; craniovertebral junction; spine

$\mathrm{C}$ ONGENITAL and acquired disorders affecting the craniovertebral junction (CVJ) can present with unique neurological manifestations. ${ }^{1}$ This is due to the underlying neuroanatomical and biomechanical properties of the region and its involvement by diverse pathological entities, including trauma, neoplasms, developmental anomalies, and vascular and degenerative conditions. ${ }^{14}$ Lack of recognition and delayed diagnosis is often associated with long-term neurological injury and morbidity.

Proatlas segment bony anomalies are a rare subset of the congenital disorders affecting the CVJ ${ }^{15}$ Neurological manifestations of this congenital disorder arise as a consequence of bony compression of the craniocervical junction. We describe a rare clinical presentation in a patient with an underlying proatlas segment bony abnormality who presented with a palatal tremor. Palatal tremor is a rare movement disorder that consists of involuntary rhythmic muscular contractions of the palatal musculature. ${ }^{21}$ In contrast to other treatments for the common causes of palatal tremor, surgical decompression of the underlying craniovertebral anomaly enabled successful resolution of the patient's neurological manifestations.

\section{Case Report}

History and Examination

The patient was a 7-year-old boy who initially presented to the pediatric neurology service for evaluation of seizure-like episodes, as described by his parents, for the preceding 6 months. These self-limiting episodes consisted of characteristic lip smacking and tongue clicking that the child made by touching his tongue to his palate. These episodes initially lasted for 15-30 seconds and were not associated with aural, post-"ictal" phases or loss of bladder/bowel continence. He also had associated ataxia and complained of frequent headaches. In addition, the parents described behavioral problems at school, with interpersonal conflicts and emotional outbursts. His parents also described loss of hand dexterity.

After evaluation by the general pediatricians and pediatric psychiatrists, he was diagnosed with Asperger's syndrome. On further evaluation by the pediatric neurologists, results of his initial imaging studies, including CT and MRI, were interpreted as normal. Electroencephalography and inpatient video electroencephalography monitor-

ABBREVIATIONS CVJ = craniovertebral junction; EPT = essential palatal tremor; GMT = Guillain-Mollaret triangle; SPT = symptomatic palatal tremor . SUBMITTED November 26, 2014. ACCEPTED January 22, 2015.

INCLUDE WHEN CITING Published online May 29, 2015; DOI: 10.3171/2015.1.PEDS14671.

DISCLOSURE The authors report no conflict of interest concerning the materials or methods used in this study or the findings specified in this paper. 
ing showed no evidence of seizure activity. He underwent genetic testing that did not reveal any underlying abnormality or syndromic condition.

A subsequent MRI study of the brain was recognized to indicate basilar invagination, and he was referred for neurosurgical evaluation. On presentation to our service, he had been experiencing these spells with increasing frequency and duration over the preceding 4-5 years. His parents described stereotypical episodes consisting of lip smacking, clucking, guttural voices, and extensor posturing with associated ataxia. Neurological examination indicated intact cranial nerve. His palate elevated symmetrically but had rhythmic clonus. Upper-extremity deep tendon reflexes showed hyperreflexia.

\section{Neuroimaging Findings}

Admission CT of the CVJ indicated proatlas segmentation abnormality with complete absence of the odontoid process (Fig. 1A). The abnormal bony segment was attached to the clivus that extended into the spinal canal, with resultant ventral cervicomedullary junction indentation (Fig. 1B, arrowhead). The MRI studies indicated a bilateral hyperintense signal within the inferior olivary nucleus (Fig. 1C, arrows). Significant ventral compression was evident (Fig. 1D). The clivus-canal angulation was significantly worse in flexion $\left(97^{\circ}\right)$ compared with extension $\left(114^{\circ}\right)$ (Fig. 1E). Tonsillar ectopia was also present. On review, these imaging findings were present in earlier imaging studies. However, radiographic progression was evident over time.

\section{Operation}

Following fiberoptic intubation and pharmacological induction of neuromuscular paralysis, intraoperative manual reduction was attempted with crown halo traction (Fig. 2A). Intraoperative CT O-arm imaging indicated irreducible ventral cervicomedullary compression and minimal improvement in cervicomedullary alignment postreduction. Hence, an anterior transpalatopharyngeal decompression of the ventral cervicomedullary junction was undertaken. Bony excision of anterior arch of the atlas, superior portion of the axis body, and midline excision of the proatlas bony segment enabled adequate ventral decompression of the medulla (Fig. 2B). Subsequently, a suboccipital bony decompression of the foramen magnum was performed. Gross atlantoaxial instability was noted intraoperatively. An Oc-C2 fixation was undertaken with an occipital plate and bilateral C-2 pars screws, supplemented with calvarial autograft. Neurophysiological monitoring was used throughout the procedure, including the manual reduction, as previously described. ${ }^{4}$

\section{Postoperative Course}

After postoperative convalescence, follow-up imaging showed adequate craniocervical decompression (Fig. 2C). Delayed dynamic radiographic imaging showed stable bony fusion (Fig. 2D). The parents reported complete resolution of the drop attacks and episodic oropharyngeal spasms. By 1-year follow-up, the child also showed significant improvement in his neurobehavioral milestones, with no further recurrences.

\section{Discussion}

Our case report highlights the neurological manifestations of CVJ disorders. Due to the anatomical juxtaposition of neural, vascular, and skeletal structures, CVJ disorders can sometimes present with unique neurological manifestations. ${ }^{12}$ We describe a rare presentation of symptomatic palatal tremor (SPT) as a consequence of a developmental anomaly affecting the craniocervical junction.

\section{Palatal Tremor}

Palatal tremor is a movement disorder characterized by rhythmic contractions of the soft palate. It often occurs in combination with similar rhythmic movements involving the face, larynx, and diaphragm. It has synonymously been identified by several terms, including palatal myoclonus and palatal myorhythmia, but was redefined as a pala-

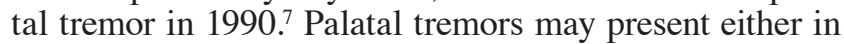
the form of idiopathic, essential palatal tremor (EPT) or as an SPT. The essential or idiopathic form develops in the absence of any structural abnormalities or CNS lesions. ${ }^{5}$ The palatal tremor often occurs in combination with a self-audible clicking sound within the ear. This occurs due to pathological activation of the tensor veli palatini muscle innervated by the trigeminal cranial nerve. The resultant repetitive opening and closure of eustachian tubes is responsible for the audible clicks. Imaging findings are typically absent.

The symptomatic or secondary form occurs as a consequence of an underlying degenerative, neoplastic, vascular, structural, or demyelinating injury involving the brainstem or cerebellum..$^{5,8,11,17,19,20}$ It consists of palatal contractions at a characteristic rhythmic frequency of 1-3 $\mathrm{Hz}$ due to involvement of the levator veli palatini muscle innervated by the glossopharyngeal cranial nerve. Ear clicks are absent and concomitant brainstem or cerebellar signs are often present due to the initial injury. Patients with SPT may also have abnormal motor learning with defective conditioning learning tasks. ${ }^{6}$

\section{Pathogenesis}

The central pathogenic mechanism involves a deafferentation injury of the inferior olivary nucleus within the dentato-rubro-olivary pathway. This functional pathway, also known as the Guillain-Mollaret triangle (GMT), comprises the contralateral dentate nucleus and the ipsilateral red nucleus and inferior olivary nucleus, respectively. ${ }^{3,7}$ The dentate nucleus projects through the superior cerebellar peduncle to the contralateral red nucleus. The efferent projections comprising this dentatorubral pathway decussate in the caudal midbrain and terminate in the rostral, parvicellular part of the red nucleus. Efferent projections descend in the ipsilateral central tegmental tract to the dorsal lamella of the inferior olivary nucleus. This feedback loop is completed by projections from the inferior olivary nucleus decussating fibers within the inferior cerebellar peduncle back to the dentate nucleus. ${ }^{8}$

The inferior olivary nucleus is hypothesized to be a central generator with an intrinsic spontaneous activity. ${ }^{3}$ In palatal tremors, the deafferentation injury arises from lesions within the first 2 parts of the GMT. The site of 

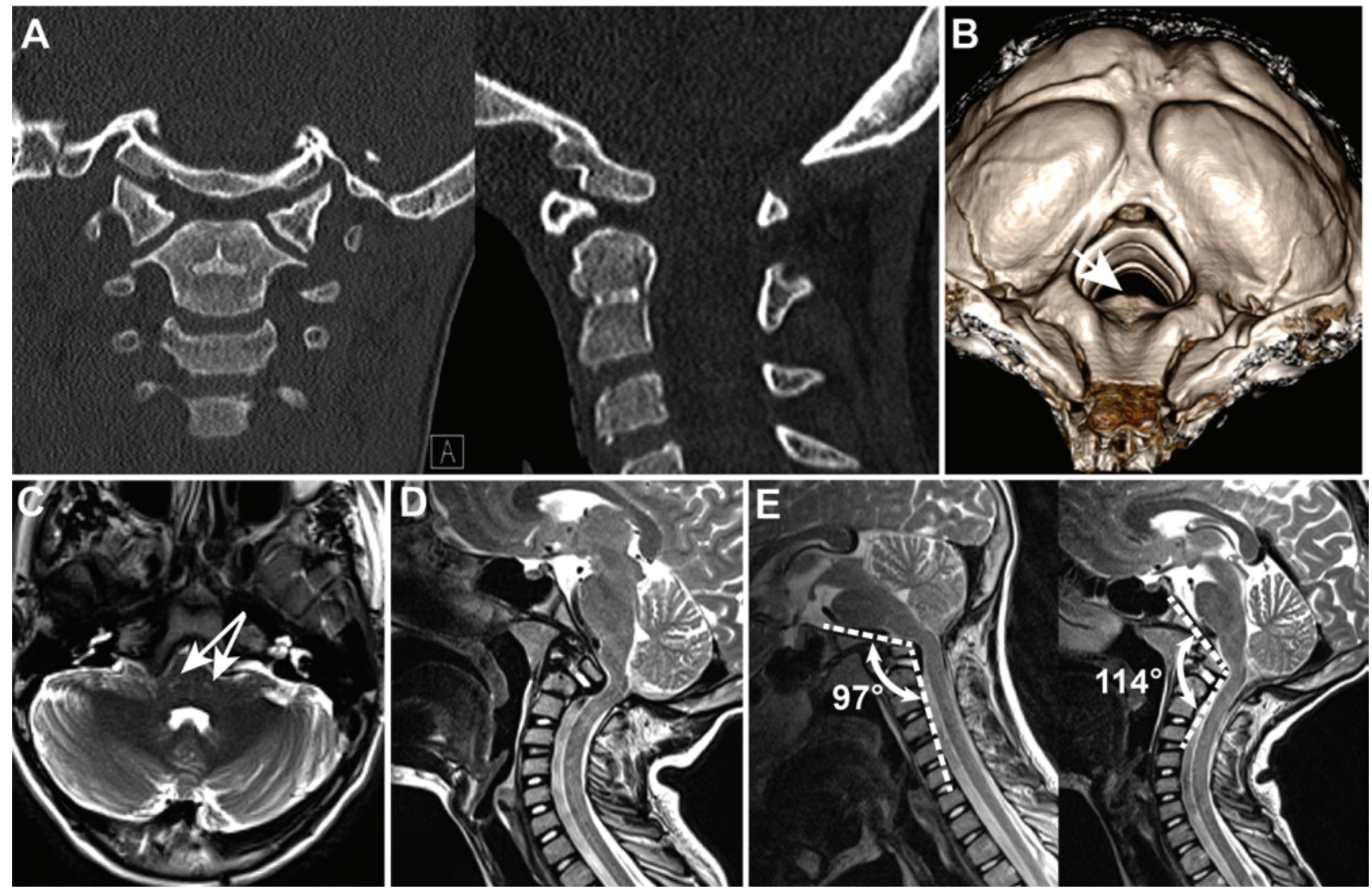

FIG. 1. A: Preoperative CT scans of the CVJ indicating proatlas segmentation abnormality with complete absence of the odontoid process (left, coronal view). The abnormal bony segment is attached to the clivus and extrudes into the spinal canal (right, sagittal view). B: A 3D reconstruction showing the proatlas remnant and the extent of spinal canal stenosis (arrowhead). C: Preoperative MRI study indicating bilateral hyperintense signal within the inferior olivary nucleus (arrows). D: Significant ventral compression of the cervicomedullary junction is evident. E: The clivus-canal angulation was significantly worse in flexion $\left(97^{\circ}\right.$, left) compared with extension $\left(114^{\circ}\right.$, right).

injury is typically ipsilateral if confined to the brainstem or contralateral if it occurs within the cerebellum. Due to the intrinsic oscillatory electrical properties of the inferior olivary nucleus, a pacemaker current is generated. This accounts for the rhythmic nature of the palatal tremor. In addition, the rhythmic pacemaker potential can also remotely modulate activity within distal spinal motor nuclei. ${ }^{7}$ Functional activation of the inferior olivary nucleus and dentate nucleus is demonstrable during the tremor periods, thereby confirming the loci. ${ }^{18}$ Tonic electromyographic activity within extremity muscles is demonstrable during palatal tremor cycles, and is most prominent in the upper rather than lower extremity and in distal versus proximal muscle groups. ${ }^{7}$ These account for the extremity and postural tremors that can be identified in patients with SPT.

The natural history of pathological and radiographic changes in relation to the onset of injury has been well described. ${ }^{8}$ Postmortem studies have helped to define the temporal profile of the associated microscopic changes. ${ }^{10}$ Neuronal hypertrophy is first demonstrable at 3 months following the lesion onset, and it peaks at 8-9 months. Following an intermediate pseudohypertrophy stage, neuronal atrophy eventually ensues. Meta-analyses have helped to delineate the temporal profile of radiographic findings in relation to the natural history of the disease. ${ }^{2,8}$ These initially include increased T2 signal intensity within 1 month of the inciting lesion, and are accompanied with a normal-sized olivary nucleus. This is followed by a phase of olivary hypertrophy due to the underlying hypertrophic olivary degeneration, with continued $\mathrm{T} 2$ signal hyperintensity, typically 6 months after the injury. Finally, olivary hypertrophy resolves in 3-4 years, with persistent $\mathrm{T} 2$ signal changes.

\section{Proatlas Segmentation Anomaly}

Proatlas bony segments are rare developmental anomalies of the CVJ. ${ }^{13}$ The CVJ develops by endochondral ossification. A cartilaginous framework that is initially formed is resorbed and replaced by ossification. Of the 42 somites, the craniocervical junction is formed by 4 occipital and the first 2 cervical sclerotomes. ${ }^{16}$ The first 2 occipital sclerotomes give rise to the basiocciput, and the third sclerotome develops into the jugular tubercles. The fourth occipital sclerotome or the proatlas segment normally develops into the anterior clival tubercle, the apical odontoid segment, parts of the foramen magnum margin, and the atlantal masses. The homeobox (Hox) and the paired box 

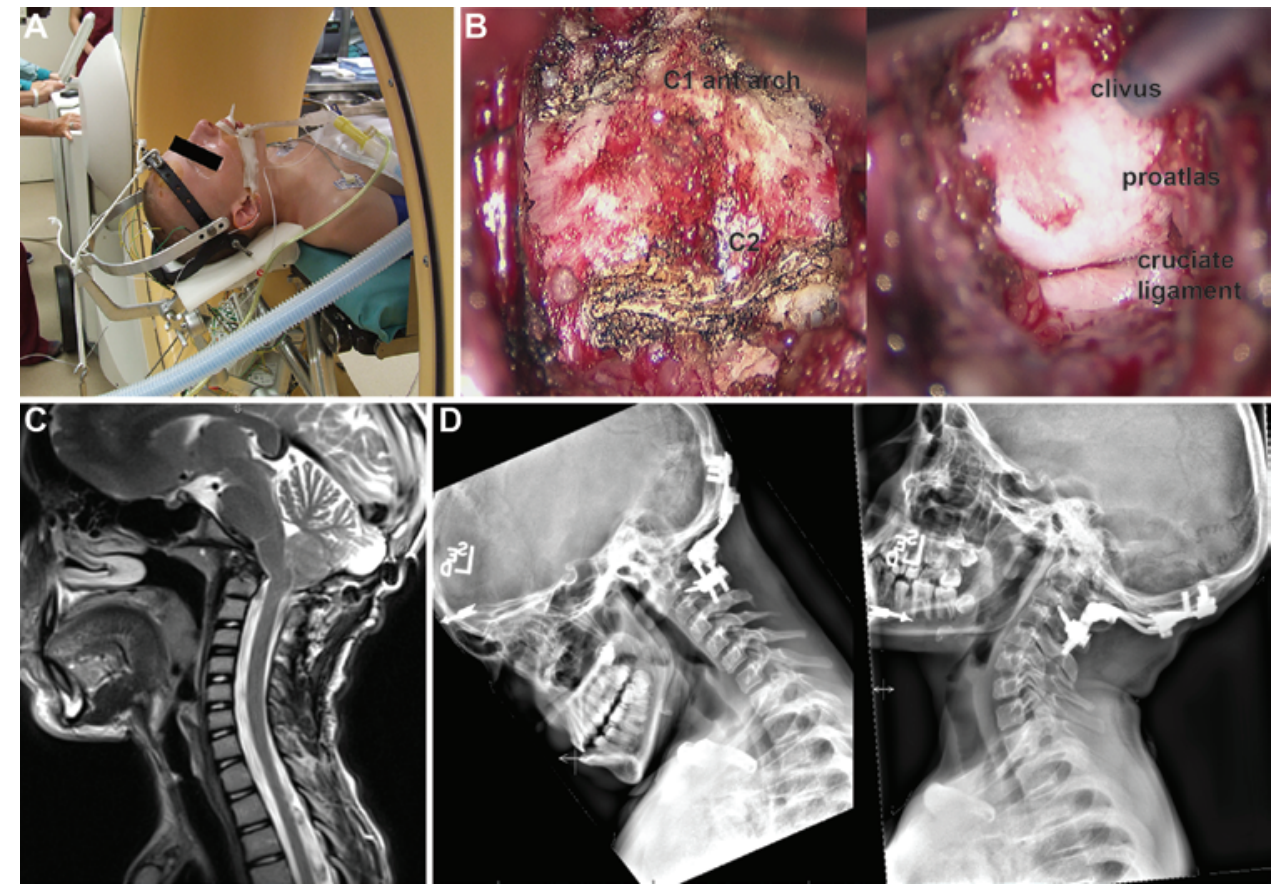

FIG. 2. A: Intraoperative photograph showing attempts at bony reduction using neuromuscular blockade and crown halo traction under neurophysiological monitoring. B: Intraoperative photographs obtained during the transoral transpalatopharyngeal approach showing the C-1 anterior arch and the C-2 vertebral body, prior to excision (left side of panel). Following this, ventral compression from the proatlas segment and the clivus was identified and removed by bony decompression (right side of panel). C: Postoperative MRI showing satisfactory ventral decompression. D: Dynamic radiographic images obtained at the 12-month follow-up interval indicating stable alignment and bony fusion. Ant = anterior.

(Pax) family of developmental genes regulate the embryonic development of the craniocervical junction. ${ }^{9}$

Postembryonic persistence of the proatlas segment can give rise to a wide range of developmental anomalies due to the integral developmental role of the proatlas segment at the CVJ. These most commonly include a prebasilar third occipital condyle, a partial regressive occipital vertebra, or an ossiculum terminale. ${ }^{15,16}$ The proatlas segment remnant in our patient represents a developmental anomaly of the hypocentrum and centrum of the fourth occipital sclerotome. The former develops into the anterior tubercle of the clivus, and the latter forms the apical dens segment.

Proatlas segments typically present within the first 2 decades of life with clinical symptoms secondary to craniocervical compression. In the largest series to date, the most common anatomical and pathological presentation consisted of ventral craniocervical compression from abnormal bony segmentation of the clivus or the occipital condyle in up to $61 \%$ of patients. ${ }^{15}$ Lateral or anterolateral compression was evident in $37 \%$ and dorsal compression in $17 \%$ of the patients. Abnormal bony development of the posterior fossa leads to reduced volume, and consequently hindbrain herniation occurs in up to one-third of patients. ${ }^{15}$ Symptom onset typically coincides with increased physical activity and as a result of trauma in up to $55 \%$ of patients. Neurological manifestations included motor deficits in up to $72 \%$ and lower cranial nerve palsies were present in $33 \%$ of patients. Neurological symptoms may also arise from vascular compression of the vertebrobasilar system from proatlas bony remnants.
Surgical treatment is directed at decompression of the abnormal bony segment. In the event of the common ventral craniocervical compression, a transoral transpalatopharyngeal approach is necessitated. This was paired with dorsal occipitocervical fusion in almost all instances..$^{15}$ Lateral compression is addressed via posterolateral or far lateral transcondylar approaches. Finally, dorsal compression is relieved by posterior approaches.

The diagnosis of SPT in our patient was based on the following reasons: first, the characteristic palatal tremor with the associated oropharyngeal muscular contractions in our patient is a principal feature of SPT. The facial spasms noted in our patient can be explained by coactivation of the facial nucleus with the levator veli palatini muscle.? Second, coexisting brainstem and cerebellar signs, typically found in patients with SPT, were also identified in our patient in the form of ataxia, myelopathic symptoms, and pendular nystagmus. Our patient also developed extremity and postural tremor that can coexist in patients with SPT due to the underlying pacemaker activity of the inferior olivary nucleus. Third, an underlying structural cause was identified in the form of a proatlas segmentation defect with associated ventral craniocervical compression. The ventral midline compression from the prosegmentation bony anomaly probably led to dysfunction of the central tegmental tract. ${ }^{7}$ This also explains the bilateral nature of symptom presentation in our patient. Fourth, the clinical symptoms resolved postoperatively following surgical treatment of the underlying bony anomaly by craniovertebral decompression and stabilization. Finally, most cases 
are identifiable by $\mathrm{T} 2$ signal hyperintensity within the inferior olivary nucleus, 7 as in our patient (Fig. 1C).

\section{Conclusions}

Our case report serves to emphasize the varied neurological presentations associated with $\mathrm{CVJ}$ disorders. Prompt recognition and diagnosis can lead to successful treatment and often neurological resolution of the underlying neurological manifestations. It also serves to highlight a rare but treatable cause for SPT in the form of a developmental CVJ disorder, with neurological recovery after surgical treatment of the underlying segmentation defect.

\section{References}

1. Benglis D, Levi AD: Neurologic findings of craniovertebral junction disease. Neurosurgery 66 (3 Suppl):13-21, 2010

2. Birbamer G, Buchberger W, Felber S, Aichner F: MR appearance of hypertrophic olivary degeneration: temporal relationships. AJNR Am J Neuroradiol 13:1501-1503, 1992

3. Borruat FX: Oculopalatal tremor: current concepts and new observations. Curr Opin Neurol 26:67-73, 2013

4. Dahdaleh NS, Dlouhy BJ, Menezes AH: Application of neuromuscular blockade and intraoperative $3 \mathrm{D}$ imaging in the reduction of basilar invagination. J Neurosurg Pediatr 9:119-124, 2012

5. Deuschl G, Mischke G, Schenck E, Schulte-Mönting J, Lücking $\mathrm{CH}$ : Symptomatic and essential rhythmic palatal myoclonus. Brain 113:1645-1672, 1990

6. Deuschl G, Toro C, Valls-Solé J, Hallett M: Symptomatic and essential palatal tremor. 3. Abnormal motor learning. J Neurol Neurosurg Psychiatry 60:520-525, 1996

7. Deuschl G, Toro C, Valls-Solé J, Zeffiro T, Zee DS, Hallett M: Symptomatic and essential palatal tremor. 1. Clinical, physiological and MRI analysis. Brain 117:775-788, 1994

8. Goyal M, Versnick E, Tuite P, Cyr JS, Kucharczyk W, Montanera W, et al: Hypertrophic olivary degeneration: metaanalysis of the temporal evolution of MR findings. AJNR Am J Neuroradiol 21:1073-1077, 2000

9. Kessel M, Balling R, Gruss P: Variations of cervical vertebrae after expression of a Hox-1.1 transgene in mice. Cell 61:301-308, 1990

10. Khoyratty F, Wilson T: The dentato-rubro-olivary tract: clinical dimension of this anatomical pathway. Case Rep Otolaryngol 2013:934386, 2013

11. Kulkarni PK, Muthane UB, Taly AB, Jayakumar PN, Shetty R, Swamy HS: Palatal tremor, progressive multiple cranial nerve palsies, and cerebellar ataxia: a case report and review of literature of palatal tremors in neurodegenerative disease. Mov Disord 14:689-693, 1999

12. Marano SR, Calica AB, Sonntag VK: Bilateral upper extremity paralysis (Bell's cruciate paralysis) from a gunshot wound to the cervicomedullary junction. Neurosurgery 18:642-644, 1986

13. Menezes AH: Craniocervical developmental anatomy and its implications. Childs Nerv Syst 24:1109-1122, 2008

14. Menezes AH: Craniovertebral junction database analysis: incidence, classification, presentation, and treatment algorithms. Childs Nerv Syst 24:1101-1108, 2008

15. Menezes AH, Fenoy KA: Remnants of occipital vertebrae: proatlas segmentation abnormalities. Neurosurgery 64:945954, 2009

16. Muhleman M, Charran O, Matusz P, Shoja MM, Tubbs RS, Loukas M: The proatlas: a comprehensive review with clinical implications. Childs Nerv Syst 28:349-356, 2012

17. Nishigaya K, Kaneko M, Nagaseki Y, Nukui H: Palatal myoclonus induced by extirpation of a cerebellar astrocytoma. Case report. J Neurosurg 88:1107-1110, 1998

18. Nitschke MF, Krüger G, Bruhn H, Klein C, Gehrking E, Wessel K, et al: Voluntary palatal tremor is associated with hyperactivation of the inferior olive: a functional magnetic resonance imaging study. Mov Disord 16:1193-1195, 2001

19. Samuel M, Torun N, Tuite PJ, Sharpe JA, Lang AE: Progressive ataxia and palatal tremor (PAPT): clinical and MRI assessment with review of palatal tremors. Brain 127:12521268,2004

20. Shioda M, Hayashi M, Takanashi J, Osawa M: Lesions in the central tegmental tract in autopsy cases of developmental brain disorders. Brain Dev 33:541-547, 2011

21. Zadikoff C, Lang AE, Klein C: The 'essentials' of essential palatal tremor: a reappraisal of the nosology. Brain 129:832-840, 2006

\section{Author Contributions}

Conception and design: Menezes. Acquisition of data: both authors. Analysis and interpretation of data: both authors. Drafting the article: both authors. Critically revising the article: both authors. Reviewed submitted version of manuscript: both authors. Approved the final version of the manuscript on behalf of both authors: Menezes. Study supervision: Menezes.

\section{Correspondence}

Arnold H. Menezes, Department of Neurosurgery, University of Iowa Hospitals and Clinics, 200 Hawkins Dr., 1824 JPP, Iowa City, IA 52242.email: arnold-menezes@uiowa.edu. 